\title{
Doubly-polarised pion photoproduction on the nucleon at MAMI
}

\author{
Susanna Costanza ${ }^{1,2}$, a for the A2 Collaboration \\ ${ }^{1}$ Dipartimento di Fisica, Università degli Studi di Pavia, I-27100 Pavia, Italy \\ ${ }^{2}$ INFN, Sezione di Pavia, I-27100 Pavia, Italy
}

\begin{abstract}
The A2 Collaboration at MAMI (Mainz) carried out new measurements of the helicity dependence of the total inclusive photo-absorption cross section and of the partial cross sections for several reaction channels on the proton and on the neutron in the photon energy region $200<E_{\gamma}<1500 \mathrm{MeV}$.

The experiments were performed at the tagged photon beam facility of the MAMI accelerator in Mainz, using circularly and linearly polarised photons on longitudinally polarised proton, deuteron and ${ }^{3} \mathrm{He}$ targets. Hadronic reaction products were detected by the large acceptance Crystal Ball-TAPS spectrometer, complemented by plastic scintillators and vertex detectors for charged particle tracking and identification.

These new, high-quality doubly-polarised pion-photoproduction data sets provide a valuable input to the study of the nucleon structure and excitation spectrum by significantly constraining the electromagnetic multipole evaluation performed by the different available partial wave analysis models. Furthermore, the helicity dependent observables provide the main ingredient for the verification of the well-known Gerasimov-Drell-Hearn (GDH) sum rule, which relates the helicity-dependent photoasborption process to the main static nucleon properties (mass, charge, spin).
\end{abstract}

\section{Introduction}

The structure of the nucleus has been a central issue of nuclear and particle physics since many years, as well as the subject of intense research, both from the theoretical and experimental point of view. The study of the excited states of the nucleon is of particular interest: they are related to the fundamental properties of the strong interaction in the same way as the excitation spectra of atoms reflect the properties of the electromagnetic interaction.

Since Quantum Chromodynamics (QCD) is nonperturbative at this energy scale, we have to rely on phenomenological quark models to interpret the experimental results. Unfortunately, this approach has limited success: at present, while there is quite an agreement between the experimental findings and model predictions in the low mass region of the nucleon excitation spectrum, there is still a pletora of higher lying states predicted by theories and not yet observed ("missing resonances").

From the experimental point of view, the reason for this mismatch can be due to data analysis relying mostly on pion scattering, that will therefore miss states that couple only weakly to $\pi N$ : it may be that the higher lying states couple preferentially to decay channels involving heavier mesons or decay via intermediate excited states [1].

In order to understand the nucleon excitation spectra, an alternative is represented by the use of electromagneticinduced reactions, like meson photoproduction: in such a way, it is possible to explore multiple meson production

\footnotetext{
a e-mail: susanna.costanza@pv.infn.it
}

states, like $\pi \pi, \pi \eta, \ldots$, and access resonances that decay mainly via intermediate excited states.

In particular, photoproduction reactions of single pseudoscalar mesons allow to measure a set of 16 polarisation observables, for every fixed value of energy and angle $(W, \theta)$. In addition to the unpolarised cross section $\sigma$, there are 3 single polarisation observables and 12 double polarisation observables, grouped in beam-target, beam-recoil nucleon and target-recoil polarisation classes (table 1). The measurement of 7 (8) properly chosen observables is necessary to yield a model-independent complete analysis $[2,3]$.

\section{The A2 Collaboration at MAMI}

Thanks to the use of polarised proton, deuteron and ${ }^{3} \mathrm{He}$ targets, the A2 Collaboration can carry out a comprehensive experimental program in order to give a better insight into the GDH sum rule on the proton and on the neutron, and to perform an accurate investigation of the properties of baryonic resonances through experiments devoted to the study of double-polarisation observables.

\subsection{The detector}

The experimental setup of the A2 Collaboration is located at the MAMI tagged photon beam facility in Mainz (Germany). The photon beam is produced via 
Table 1. Polarisation observables.

\begin{tabular}{|c|c|c|c|c|c|c|c|c|c|c|}
\hline \multirow[t]{2}{*}{$\begin{array}{l}\text { Photon } \\
\text { polarisation }\end{array}$} & & \multicolumn{2}{|c|}{$\begin{array}{l}\text { Target } \\
\text { polarisation }\end{array}$} & \multicolumn{3}{|c|}{$\begin{array}{l}\text { Recoil nucleon } \\
\text { polarisation }\end{array}$} & \multicolumn{4}{|c|}{$\begin{array}{l}\text { Target and recoil } \\
\text { polarisation }\end{array}$} \\
\hline & & $\begin{array}{ll}X & Y\end{array}$ & $\mathrm{Z}_{\text {(beam) }}$ & $\mathrm{X}^{\prime}$ & $\mathrm{Y}^{\prime}$ & $Z^{\prime}$ & $\begin{array}{l}X^{\prime} \\
X\end{array}$ & $\begin{array}{l}X^{\prime} \\
Z\end{array}$ & $\begin{array}{l}\mathrm{Z}^{\prime} \\
\mathrm{X}\end{array}$ & $\begin{array}{l}\mathrm{Z}^{\prime} \\
\mathrm{Z}\end{array}$ \\
\hline $\begin{array}{l}\text { unpolarised } \\
\text { linear } \\
\text { circular }\end{array}$ & $\begin{array}{l}\sigma \\
-\Sigma \\
-\end{array}$ & $\begin{array}{cc}- & \mathrm{T} \\
\mathrm{H} & (-\mathrm{P}) \\
\mathrm{F} & -\end{array}$ & $\begin{array}{l}- \\
-G \\
-E\end{array}$ & $\begin{array}{l}- \\
\mathrm{O}_{X} \\
\mathrm{C}_{X}\end{array}$ & $\begin{array}{l}\mathrm{P} \\
(-\mathrm{T}) \\
-\end{array}$ & $\begin{array}{l}- \\
\mathrm{O}_{Z^{\prime}} \\
\mathrm{C}_{Z^{\prime}}\end{array}$ & $\begin{array}{c}\mathrm{T}_{X^{\prime}} \\
\left(-\mathrm{L}_{Z^{\prime}}\right) \\
-\end{array}$ & $\begin{array}{c}\mathrm{L}_{X^{\prime}} \\
\left(\mathrm{T}_{Z^{\prime}}\right) \\
-\end{array}$ & $\begin{array}{c}\mathrm{T}_{Z^{\prime}} \\
\left(\mathrm{L}_{X^{\prime}}\right) \\
-\end{array}$ & $\begin{array}{c}\mathrm{L}_{Z^{\prime}} \\
\left(-\mathrm{T}_{X^{\prime}}\right) \\
-\end{array}$ \\
\hline
\end{tabular}
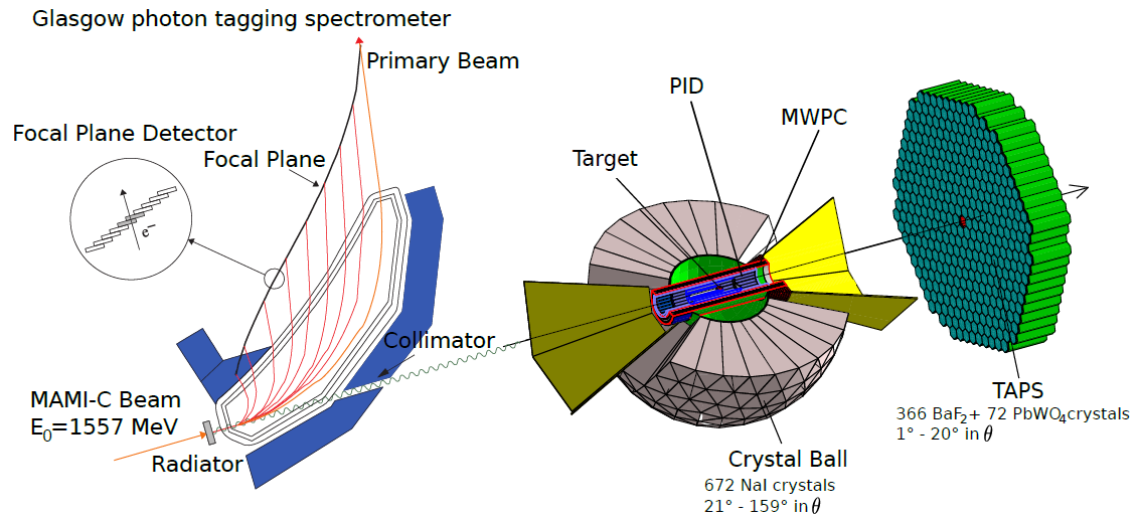

Figure 1. Schematic view of the A2 experimental setup.

bremsstrahlung of an electron beam of $1557 \mathrm{MeV}$ provided by the MAMI accelerator, and polarised up to $\sim 75$ $78 \%$. The photons are then tagged using the GlasgowMainz magnetic spectrometer, which has an energy resolution of $\sim 2 \mathrm{MeV}$.

The A2 apparatus can make use of different targets: for the experiments described in the following, a frozen-spin butanol $\left(\mathrm{C}_{4} \mathrm{H}_{9} \mathrm{OH}\right)$ [4] and deuterated butanol $\left(\mathrm{C}_{4} \mathrm{OD}_{10}\right)$ targets, and a high pressure ${ }^{3} \mathrm{He}$ gas target have been used. Concerning the butanol target, the $2 \mathrm{~cm}$ long target cell was located in a ${ }^{3} \mathrm{He} /{ }^{4} \mathrm{He}$ dilution refrigerator and the target nucleons were polarised via Dynamic Nuclear Polarisation (DNP) [5] to an initial polarisation degree up to $90 \%$. During the data taking, a magnetic field of $0.68 \mathrm{~T}$ together with the $25 \mathrm{mK}$ temperature provided by the dilution refrigerator ensured long relaxation times (up to 2000 h) before repolarisation was required. Since only the hydrogen nuclei of the butanol can be polarised, a carbon foam target was used in a dedicated beamtime to study background contributions from unpolarised carbon nuclei. In the case of the frozen-spin target using deuterated butanol, a starting value of target polarisation up to $70 \%$, with a relaxation time of $\sim 200$ hours, was obtained, thanks to a new doping material [6]. Also in the case of gaseous ${ }^{3} \mathrm{He}$ an initial polarisation value up to $70 \%$ was obtained, via the metastability exchange optical pumping (MEOP [7]) method, with a total relaxation time of $\sim 20$ hours.

The target cell is sitting inside the central detector system and surrounded by the Particle IDentification (PID) detector, made of plastic scintillators. The PID is enclosed by the Multi-Wire Proportional Chambers (MWPCs), used to discriminate between charged and neutral particles. The MWPCs are complemented by the Crystal Ball (CB) NaI spectrometer, a large solid angle, highly segmented photon and hadron spectrometer. By combining the information provided by these three detectors, precise energy measurements, as well as angle and particle identification in the azimuthal $(\phi)$ and polar $(\theta)$ angular regions from $0^{\circ}$ to $360^{\circ}$ and from $21^{\circ}$ to $159^{\circ}$, respectively, are obtained.

\section{Double polarisation observables $\mathrm{G}$ and E}

The double-polarisation observables $\mathrm{G}$ and $\mathrm{E}$, as shown in table 1 , require the polarisation of both the photon beam and the target.

They are derived from the differential cross section for pseudo-scalar meson photoproduction; in the case of elliptically polarised photons in combination with a longitudinally polarised target, the cross section is given by:

$$
\begin{aligned}
\frac{d \sigma}{d \Omega}(\theta, \phi)=\frac{d \sigma}{d \Omega_{0}}(\theta) & {\left[1-P_{\text {lin }} \Sigma \cos (2(\alpha-\phi))\right.} \\
& -P_{z}\left(-P_{\text {lin }} G \sin (2(\alpha-\phi))+P_{\text {circ }} E\right] .
\end{aligned}
$$

The $E$ observable is extracted from eq. 1 by integrating over $\phi$ :

$$
\begin{aligned}
& \left.N_{B}\right|_{ \pm \alpha} ^{ \pm P_{z}}(\theta)=N_{B}(\theta) \cdot\left[1-P_{\text {circ }} P_{z} E\right] \rightarrow \\
& E=\frac{\sigma^{1 / 2}-\sigma^{3 / 2}}{\sigma^{1 / 2}+\sigma^{3 / 2}}=\frac{N_{B}^{1 / 2}-N_{B}^{3 / 2}}{N_{B}^{1 / 2}+N_{B}^{3 / 2}} \cdot \frac{1}{d} \cdot \frac{1}{P_{\text {circ }} P_{z}},
\end{aligned}
$$


where $N_{B}^{1 / 2}$ and $N_{B}^{3 / 2}$ are the helicity-dependent count rates, $d$ is the dilution factor and $P_{\text {circ }} P_{z}$ are the degrees of target and beam polarisation.

Since the experiment was performed with a butanol target, the count rates take into account the contribution of both the polarisable free protons in hydrogen nuclei and the unpolarised bound nucleons in carbon and oxygen nuclei. The amount of polarisable free protons in the data is specified by the dilution factor $d\left(d=N_{\text {free }} /\left(N_{\text {free }}+N_{\text {bound }}\right)\right)$, which was determined during a dedicated data taking using a carbon foam target. $d$ was obtained as $d=1-s$. $N_{C} / N_{B}$, where the coplanarity distribution of the carbon data $\left(N_{C}\right)$ is scaled to the one of the butanol data $\left(N_{B}\right)$. In the formula, $s$ takes into account possible differences in acceptance, photon flux and target density during the carbon and butanol beamtimes.

In the same way as $E$, the observable $G$ is obtained by integrating Eq. 1 over all possible helicity states:

$$
\begin{aligned}
\left.N_{B}\right|_{ \pm \alpha} ^{ \pm P_{z}}(\theta, \phi)=N_{B}(\theta) \cdot[ & 1-P_{l i n} \Sigma_{B} \cos (2(\alpha-\phi)) \\
+ & \left.d P_{\text {lin }} P_{z} G \sin (2(\alpha-\phi))\right] .
\end{aligned}
$$

\subsection{Experimental results}

The double polarisation observables $\mathrm{G}$ and $\mathrm{E}$ were extracted from the data according to Eqs. 2 and 3 as a function of the beam energy $E_{\gamma}$ for the entire angular range $-1 \leq \cos \theta_{\pi} \leq 1$.

Figures 2 and 3 show preliminary results for the photoproduction reaction $\vec{\gamma} \vec{p} \rightarrow p \pi^{0}$. The results are compared to data from the CBELSA/TAPS collaboration ([8] for the $G$ observable and [9] for E) and to different partial wave analyses: the agreement with the existing data and mostly with the different PWA models, especially with the BnGa201402 PWA solution, is shown for both the double polarisation observables.

\section{The GDH sum rule}

Since sum rules connect information from all energies to fundamental parameters of the interaction models of interest, they are important tools to study the excited states of the nucleons.

One of these sum rules is the well known GerasimovDrell-Hearn (GDH) sum rule [13, 14], which relates the nucleon anomalous magnetic moment (AMM) $\kappa$, the spin $S$ and the mass $M$ of a nucleon to the integral over the weighted helicity difference of the total absorption cross section for circularly polarised photons on a longitudinally polarised nucleon target:

$$
I_{G D H}=\int_{v_{t h}}^{\infty} \frac{\sigma_{p}-\sigma_{a}}{v} d v=4 \pi^{2} \kappa^{2} \frac{e^{2}}{M^{2}} S,
$$

where $v$ is the photon energy, and $\sigma_{p}$ and $\sigma_{a}$ denote the total absorption cross section for parallel and antiparallel orientation of photon and particle spins, respectively. The lower limit of the integral, $v_{t h}$, corresponds to pion production and photodisintegration threshold for a nucleonic and nuclear target, respectively.

The GDH sum rule is derived from very general fundamental physical principles, like Lorentz invariance, the optical and the low energy theorems. Therefore, its verification is of particular interest in the understanding of our knowledge of the $\gamma-N$ interaction, as well as of the physics of strongly interacting systems and of the existing photo-reaction models. Furthermore, by studying the helicity dependent $N \pi$ channels, it is possible to test multipole models, to access new observables and to study the baryonic resonances.

Thanks to the use of polarised proton, deuteron and ${ }^{3} \mathrm{He}$ targets, the A2 Collaboration can study all the $\gamma N \rightarrow$ $N \pi(\pi)$ partial channels, as well as the total cross sections, for the proton and the neutron. It can also give a better insight into the GDH sum rule and perform an accurate investigation of the baryonic resonance properties.

\subsection{Experimental results}

The experiments were carried out by using circularly polarised photons obtained via bremsstrahlung of longitudinally polarised electrons of about $75 \%$.

Different targets have been used: for the proton studies, the same frozen-spin butanol data collected for the E and $\mathrm{G}$ double polarisation observables were used.

To compensate the lack of free neutron targets, polarised deuteron and high pressure ${ }^{3} \mathrm{He}$ gas targets were used as polarised neutron sources, as described in section 2.1.

\subsubsection{Results on the proton and deuteron}

Fig. 4 (blue circles) shows the preliminary results of the helicity dependence of the total inclusive cross section $\Delta \sigma_{t o t}=\left(\sigma_{a}-\sigma_{p}\right)$ on the proton (a) and on the neutron (b). The A2 results are compared to those obtained by the GDH Collaboration $[15,16]$ (red circles), showing a good agreement within the errors.

The results for the partial channels $\gamma p \rightarrow \pi^{0} X$ and $\gamma d \rightarrow$ $\pi^{0} X$ are depicted in Fig. 5: also in these plots, the A2 data (blue circles) are compared with good agreement to the GDH Collaboration results. Since the GDH data were collected only in the $\Delta$-resonance region, it is therefore clear that the A 2 recent results can provide a useful contribution in an energy region where no other data are available.

Our experimental results are also compared to the predictions of the MAID multipole analysis: the discrepancy with the data is due to the fact that the model is a free nucleon estimation, therefore it does not take into account the nuclear effects inside the deuteron target (Fig. 5b).

\subsubsection{Results on ${ }^{3} \mathrm{He}$}

Figure 6 shows the unpolarised and helicity-dependent total cross sections for the partial channels $\gamma^{3} \mathrm{He} \rightarrow \pi^{0} X$ and $\gamma^{3} \mathrm{He} \rightarrow \pi^{ \pm} X$ in the energy region $200<E_{\gamma}<500$ $\mathrm{MeV}$ [17].

Here the data are compared to the prediction of the FixArenhövel (FA) model (red line), which is an extention of 


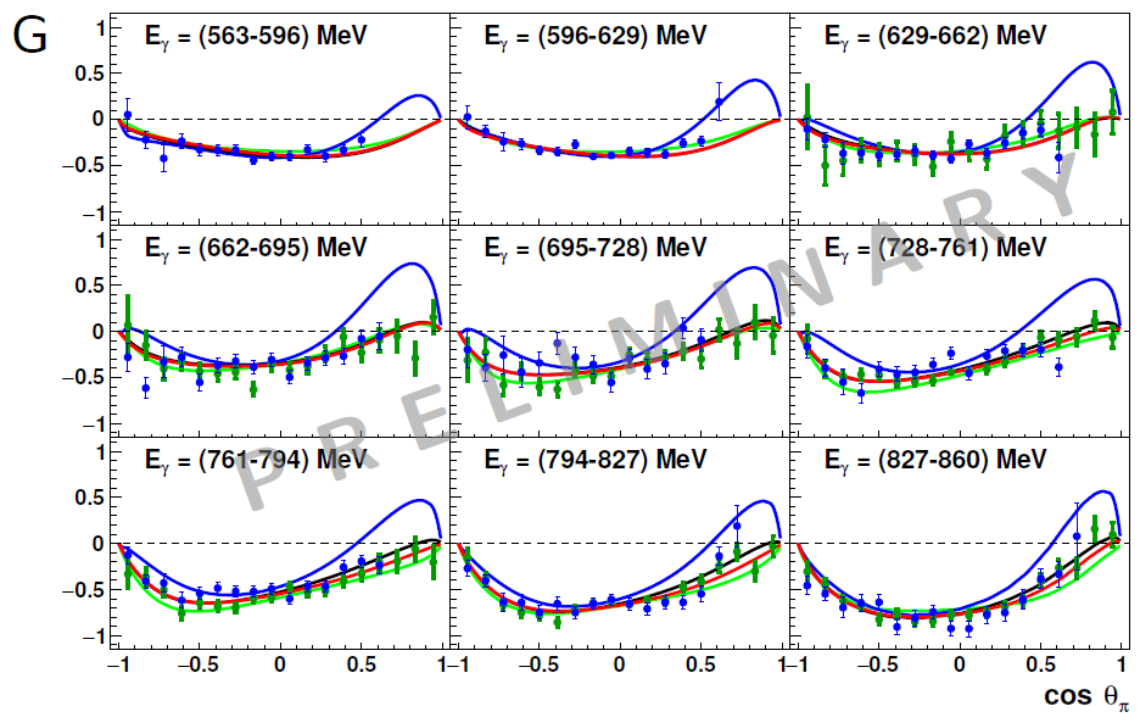

Figure 2. Results for $\mathrm{G}$ in $\pi^{0}$-photoproduction for selected energy bins, from $560 \mathrm{MeV}$ to $860 \mathrm{MeV}$. The experimental results from A2 (blue circles) are compared to the CBELSA/TAPS data (green circles) [8], to PWA fits (BnGa_2014_02 [10], red line and BnGa_2014_01, black line) and PWA predictions from MAID2007 [11] (green line) and SAID-CM12 [12] (blue line).

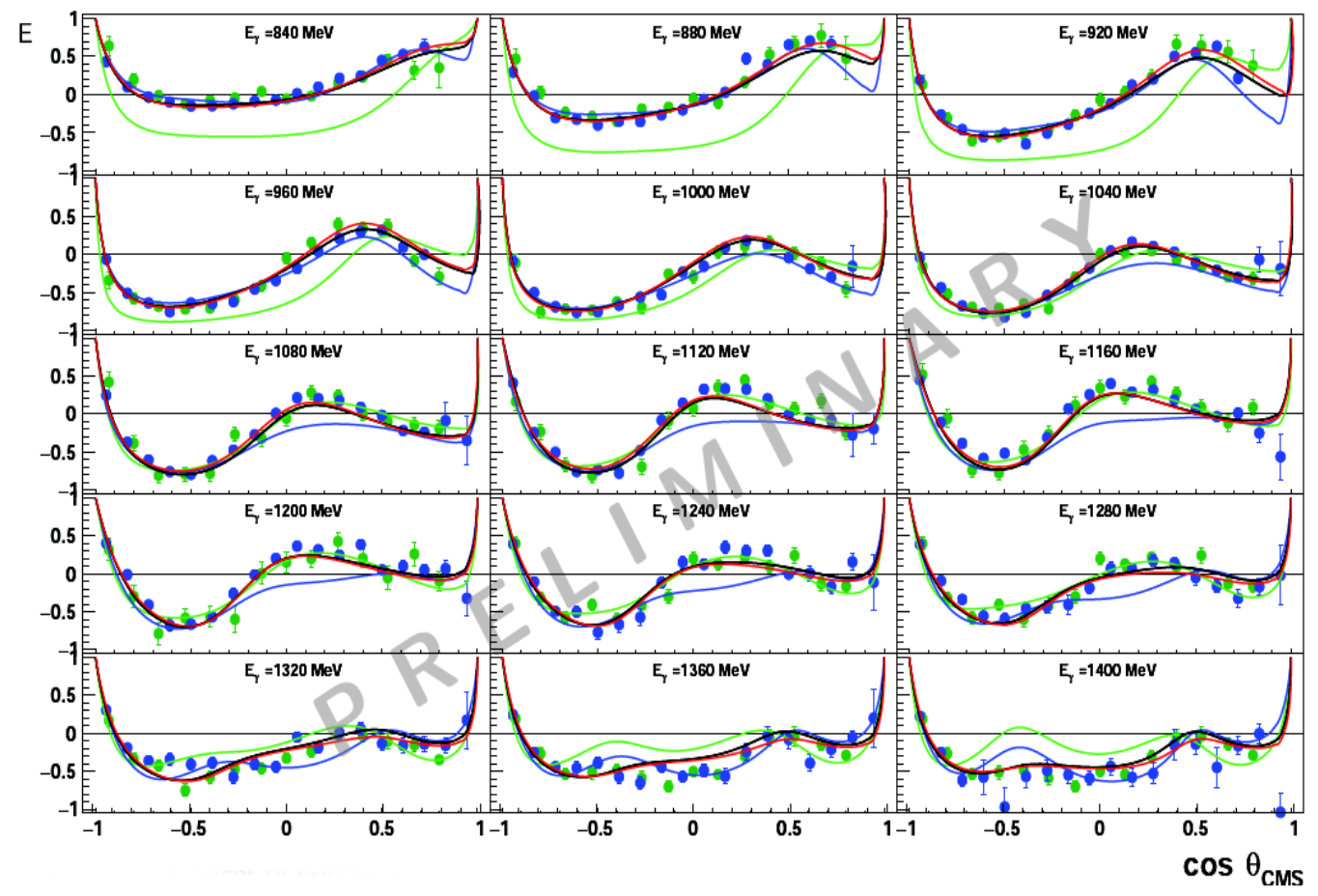

Figure 3. Results for $\mathrm{E}$ in $\pi^{0}$-photoproduction for selected energy bins from $840 \mathrm{MeV}$ to $1400 \mathrm{MeV}$. The experimental results from A2 (blue circles) are compared to the CBELSA/TAPS data (green circles) [9], to PWA fits (BnGa_2014_02 [10], red line and BnGa_2014_01, black line) and PWA predictions from MAID2007 [11] (green line) and SAID-CM12 [12] (blue line). 

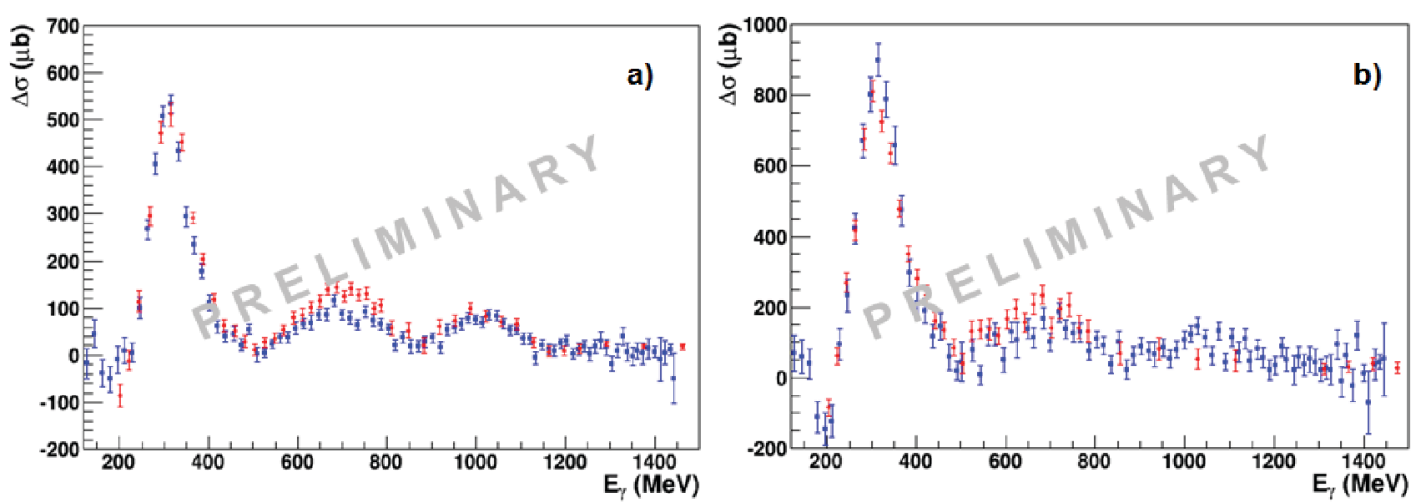

Figure 4. Helicity dependent total inclusive cross section for the $\gamma p \rightarrow X$ (a) and $\gamma d \rightarrow X$ channels (b). The A2 results (blue circles) are compared to the results from the GDH Collaboration (red circles) $[15,16]$.
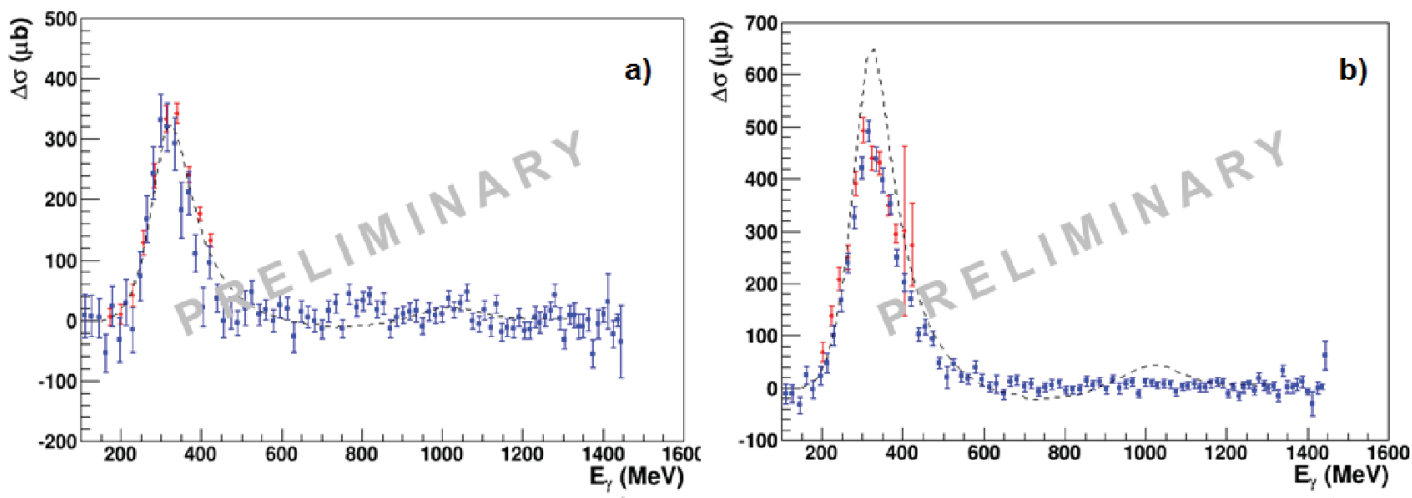

Figure 5. Helicity dependent total cross section for the semi-exclusive channels $\gamma p \rightarrow \pi^{0} X$ (a) and $\gamma d \rightarrow \pi^{0} X$ channels (b). The A2 results (blue circles) are compared to the results from the GDH Collaboration (red circles) $[15,16]$ and to the MAID model (dashed line).

the model on the deuteron [18]. In addition, the A2 results are compared to a simple plane-wave impulse approximation (PWIA) model (blue line), where the cross sections are evaluated as an incoherent sum of quasi-free single nucleon contributions, determined using the MAID multipole analysis and the momentum distribution of the nucleons inside ${ }^{3} \mathrm{He}$ as parametrised in [19]. The difference between the two models, i.e. the role of nuclear effects, results in damping and broadening the peak corresponding to the $\Delta$ resonance excitation.

In the unpolarised case, for the neutral partial channel the FA model is in good agreement with our data for $E_{\gamma} \geq$ $250 \mathrm{MeV}$, while it underestimates them at lower photon energies, where the coherent $\gamma^{3} \mathrm{He} \rightarrow \pi^{0}{ }^{3} \mathrm{He}$ reactions is expected to play a dominant role. On the contrary, the FA model describes the measured cross section of the charged channel less well and for $E_{\gamma} \geq 350 \mathrm{MeV}$ the PWIA model does better.

As in the unpolarised case, in the helicity dependent cross section case, the FA model describes the $\gamma^{3} \mathrm{He} \rightarrow \pi^{0} \mathrm{X}$ data at higher photon energies while there is less agreement with the experimental data of the charged partial channel. The PWIA model, instead, reproduces the data at higher $E_{\gamma}$ values for both reactions reasonably well.

Furthermore, to understand the origin of the observed discrepancies between the experimental models and the data, an analysis of both the unpolarised and polarised differential cross sections for the $\gamma^{3} \mathrm{He} \rightarrow \pi^{0} X$ and $\gamma^{3} \mathrm{He} \rightarrow \pi^{ \pm} X$ has been performed: the results can be found in [20].

\section{Conclusions}

Double polarised pion photoproduction experiments are a useful tool for the understanding of the nucleon excitation spectra.

The A2 Collaboration at MAMI has performed the first simultaneous measurement of the double polarisation observables $\mathrm{E}$ and $\mathrm{G}$, by using a longitudinally polarised electron beam incident on a diamond radiator and a longitudinally polarised frozen-spin target. The preliminary 

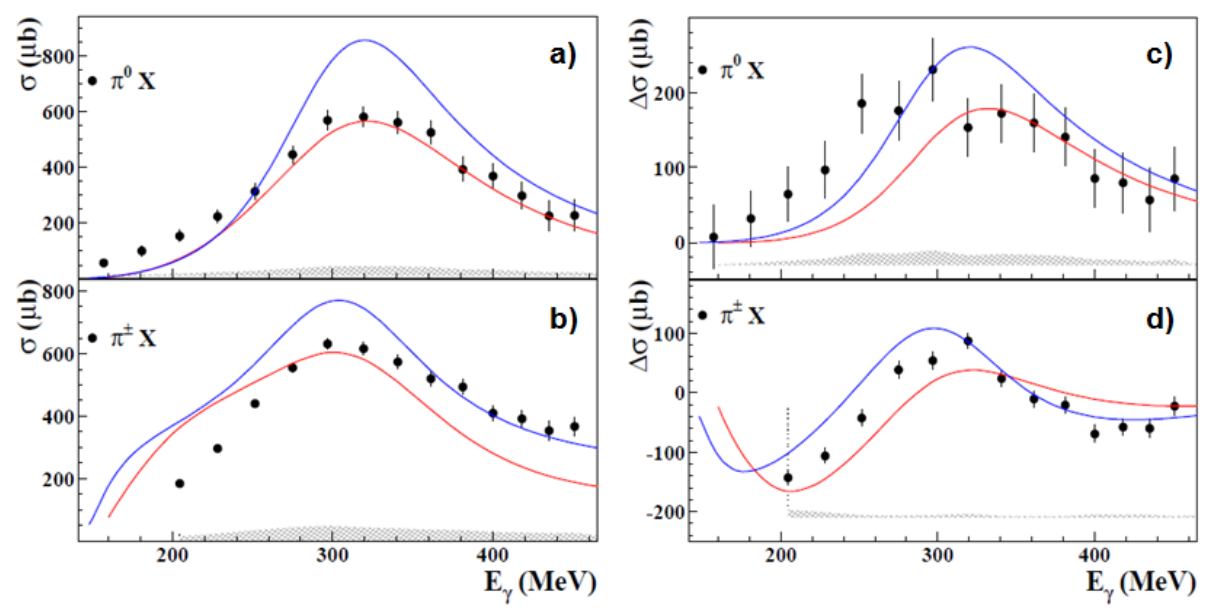

Figure 6. Unpolarised (left) and polarised (right) total cross section for $\gamma^{3} H e \rightarrow \pi^{0} X$ (a and c) and $\gamma^{3} H e \rightarrow \pi^{ \pm} X$ (b and d) channels. The error bars are statistical and the hatched bands show the systematic uncertainties. The measured cross sections are compared to the FA model (red line) and to our PWIA model (blue line).

results available up to $E_{\gamma}=1500 \mathrm{MeV}$ are in good agreement with the existing data from the CBELSA/TAPS Collaboration and provide an original contribution in the $\Delta(1232)$ resonance region.

The experimental data obtained from these experiments can be used also to study the Gerasimov-Drell-Hearn sum rule. Furthermore, thanks to the use of polarised deuteron and ${ }^{3} \mathrm{He}$ targets, the A2 Collaboration has carried out a comprehensive experimental program to study the GDH sum rule not only on the proton, but also on the neutron and on ${ }^{3} \mathrm{He}$.

The new precise results on double-polarisation measurements of the total and differential cross sections for the partial $\gamma N \rightarrow \pi X$ channels on the proton and on the neutron, obtained by the A2 Collaboration, are compared to the existing model predictions and to the few, available results. These new data are significantly increasing the available statistics, especially on the neutron, thus providing an important testing ground for all existing models.

Moreover, the results obtained on ${ }^{3} \mathrm{He}$ give information not only on the GDH integral on the neutron, but also on the ${ }^{3} \mathrm{He}$ nuclear structure and allow an investigation of the nucleon properties inside this nucleus.

These unprecedented data have proved the feasibility of the use of polarised ${ }^{3} \mathrm{He}$ gas target to check the GDH sum rule on the neutron.

\section{References}

[1] B. Krusche and S. Schadmand, arXiv:nuclex/0306023v1
[2] I. S. Barker, A. Donnachie, J. K. Storrow, Nucl. Phys. B95, 347 (1975)

[3] W.-T. Chiang and F. Tabakin, Phys. Rev. C 55, 2054 (1997)

[4] D. Goertz et al., Proceedings of PSTP205, PoS(PSTP2015)009 (2016)

[5] D.G. Crabb and W. Meyer, Annu. Rev. Nucl. Part. Sci. 47, 67 (1997)

[6] S. Goertx et al., Nucl. Instr. Meth. A 526, 43 (2004)

[7] F. Colegrove et al., Phys. Rev. Lett. 132, 2561 (1963)

[8] A. Thiel et al., Phys. Rev. Lett. 109, 102001 (2012)

[9] M. Gottschall et a., Phys. Rev. Lett. 112, 012003 (2014)

[10] E. Gutz et al., Eur. Phys. J. A 50, 74 (2014)

[11] D. Drechsel, S.S. Kamalov and L. Tiator, Eur. Phys. J. A 34, 69 (2007)

[12] R.L. Workman, M.W. Paris, W.J. Briscoe, I.I. Strakovsky, Phys. Rev. C 86, 015202 (2012)

[13] S. B. Gerasimov, Sov. J. Nucl. Phys. 2, 430 (1966)

[14] S. D. Drell and A. C. Hearn, Phys. Rev. 96, 1428 (1966)

[15] J. Ahrens et al.,Phys. Lett. B 672, 328 (2009)

[16] H. Dutz et al., Phys. Rev. Lett. 94, 162001 (2005)

[17] P. Aguar Bartolomè et al., Phys. Lett. B 723 (2013) 71-77

[18] H. Arenhövel, A. Fix, Phys. Rev. C 72 (2005) 064004

[19] R. Schiavilla, C. Pandharipande, Nucl. Phys. A 449 (1986) 219

[20] S. Costanza et al., Eur. Phys. J. A 50173 (2014) 